\section{IDEA: an Integrated Method of Decision Making in Conceptual Design}

\author{
Andrew Masur \\ Ryerson University \\ Filippo A. Salustri, PhD, PEng \\ Ryerson University
}

Abstract

The conceptual design process is one of the most important and confusing in engineering design. The Integrated Design Exploration and Analysis (IDEA) process was created to help improve conceptual design practices in the with a supporting soffware interface, was developed to rectify these weaknesses. The interface was written in the open source program Compendium. Three multi-disciplinary case studies were conducted to validate the process. The use of IDEA led to more and higher quality design concepts.

\section{Introduction}

In our modern technological society the evidence of engineering is everywhere. From cars to computers, almost everything we can see has been touched by an
engineer. With this heavy reliance on engineering it engineer. With this heavy reliance on engineering it
would seem logical that most people would have at would seem logical that most people would have at
least a cursory understanding of what it is exactly that engineers so, yet that is not the case. Many people believe that engineering is nothing more than taking advantage of a brief moment of inspiration. While these moments are important, much of the work commonly undertaken in engineering is of a far more structured and orderly nature. Most engineering work deficiencies that have been identified in existing products, or to fill some societal need. Regardless of the type of problem, the process for finding the solution is very similar throughout industry.

The engineering process involves several phases. The first of these is the problem exploration and conceptual design phase. In this part of the design design problem and then generate some initial solutions. This can be one of the most enjoyable parts of the design process since there are few limits on the types of solutions which can be proposed and imagination runs at a very high level. However, conceptual design can also be a very confusing available to designers.

Figure 1: Various engineering processes [1]

The next question that people typically ask is if the engineering process is divided into all of these important to the final design? While all of the mos phases are vital for a succesful product, a strong case can be made for selecting conceptual design as the most critical to the final design. The main reason for his importance is the fact that the different phases of all of the subsequent steps. Because it is the firs phase, the results of conceptual design affect all of the remaining phases. The choices made in conceptu project, and poor choices there often lead to failed or substantively suboptimal products (e.g. higher cos lower reliability, longer development times). In some cases, the problems caused by the selection of suboptimal concept are so extreme that the projec itself is cancelled to minimize the losses to the during the conceptual design phase in order to increse the chances of selecting the best concept.

\section{Current Techniques in Conceptual} Design

Concept design analysis is needed to make his goal is that the conceptel design phase rarely hos enough information available to conduct a rigorous analysis. However, there are a number of techniques available that are commonly used in industry to assist in design decision-making. Many of these techniques are also usefful during the conceptual design phase. This section will The first task during conceptual design is to gain a better understanding of the problem. It is difficult to provide a reasonable answer when one does not know the question, so the first task of engineers determining that question

There are various techniques to do this. The firs one involves interviewing users to determine the
needs. Originally proposed by Ralph L. Keeney [2] this technique involves the use of personalized interviews, group interviews, and mass-distributed electronic forms to determine the needs of customers. Keeney feels that by determining the needs of average users, designers are berter able to determine whe features and characteristics should be present in the Another popular technique for gaining a better understanding of a design problem is known as trade space analysis. A trade space helps designer qualitatively "trade off" different criteria for the sake he design process are highly interconnected. The

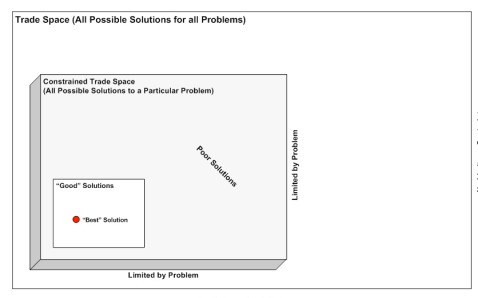

Figure 2: Trade space representatio

The large area in Figure 2 represents the total trade space, a construct that contains all possible solution to all possible design problems. The best solution to particular design problem is in this space, but is almos infinite in size Clearly, athe trade space required; this is where trade space analysis becomes useful. Through an exploration of the design problem, engineers can determine the limitations on possible designs.

These limitations define a sub-space, represented in Figure 2 by the raised grey region. This sub-space and contains all possible solutions to that problem. While this is a big improvement over the total trade space, the sub-space can still contain a vast number o Iternatives, making selection of the best one difficult. The solution is to continue analyzing the problen through brainstorming sessions, interviews with user analysis of the feasibility of options. The use of these techniques will allow for a greater understanding of the design problem and help to reduce the size of the trade space until it becomes a relatively simple task of identifying the "best" solution to the problem. While trade space exploration is meaningful an beneficial, the design team will have to make design problem.

Again, there are a number of tools available to designers to assist with this task. One of the simples is known as pairwise comparison. One of the mos common types of pairwise comparison is known as the drop and re-vote (D\&R) method. In D\&R, the concepts in a weak order. an ordint ank wing level of preference [3]. The weak orders are the "choose the best of the best" or "avoid the worst of the worst" [3]), and the most poorly ranked concepts are 
dropped from further consideration. The process is then repeated until only one alternative remains.

Like most engineering decision-making lechniques, this method violates one or more axioms
of what is known as Arrow's Impossibility Theorem, which is a set of rules (axioms) for creating a fair voting procedure [3]. In this case, a D\&R pairwise comparison breaks the Independence of Irrelevant Alternatives (IIA) axiom [3]. As a result, the ranking of alternatives changes whenever the lowest-ranked temovil of the lowest ranked item should have net affect the ranking of the other alternatives. D\&R is particularly sensitive to rank reversals of this sort because of its iterative nature.

An alternative pairwise comparison method uses what is known as the pairwise comparison chart, which is a graphical technique of pairwise identical [3] to the well-known Borda Count. Like D\&R, the Borda Count also violates the IIA axiom; however, unlike D\&R, the Borda Count is insensitive to the effects of breaking the axiom. This is because a Borda Count can be completed in one iteration and without discarding any information, so rank reversals more robust for engineering decision making. The pairwise comparison chart method shares these strengths while also allowing the design team to calculate the level of preference between the items under comparison. These attributes make the pairwise comparison chart ideal for choosing between relatively When there are multiple criteria, however,

irwise comparison chart is not enough. The solution to this problem is to use a decision matrix [4]. It is particularly useful when choosing amongst a number of different concepts whose performance is being compared by a number of different criteria. Typically, a standardized ranking scale is used to rate how well characteristics. An example of a completed decision matrix for a fictional garden tool is given in Figure 3.

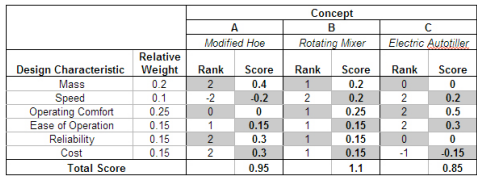

Figure 3: Completed decision matrix

The matrix shows the comparison of three different Each concept is judged against standardized set of design characteristics (derived 列 (he concepts meaningful comparisons to be made since they are all being measured against a common baseline.

The left side of the matrix also lists the relative weights of the design characteristics. Since the haracteristics are usually not equally important in secific design problem, the capacity to make such Each of the concepts is roted using a stand cale. This example uses a -2 to +2 scale that commonly used in the automotive industry. A -2 means that a concept is very poor at achieving a given haracteristic; a +2 means that a concept achieves it exceptionally well. Many other scales are possible. Simple Additive Weighting (SAW) function with the following form, where $w_{i}$ is the elative weight of a particular design characteristic an $R_{i}$ represents the rating for that characteristic for the current concept as assigned by the design team.

$$
\text { score }=\sum_{i=1}^{n} w_{i} R_{i}
$$

The concept with the highest total score best meet the given design characteristics and is most likely the test concept. While this is a very simple additive

\section{The IDEA Process}

The techniques discussed above have severa weaknesses. First, while each technique is very good a parte while trade space analysis is an exceller tool for gaining a better understanding of the problen and possibly generating concepts, it includes no real facilities to assist designers with making desig decisions. At best, it will present a limited number of suitable concepts, yet it is up on the design team matrix is it does not really help with the initial generctions, those concepts. Furthermore, the techniques really $d$ nothing to guide designers during conceptual design. The goal of the Integrated Design Exploration and Analysis (IDEA) process is to address these problem. DEA is a collecition of tools and methods intended

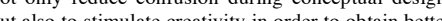

designs. IDEA provides a flexible structure for executing conceptual design. It brings togethe che the method. It lets designers focus on cognitive tasks rather than on the "administrative" side of managing the process of conceptual design. The IDEA process ollows a standardized procedure for all problems as can be seen in Figure 4 .

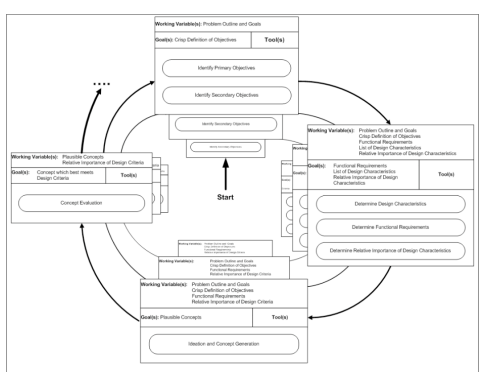

Figure 4: IDEA overall outline

As can be seen in Figure 4, the IDEA process consists of four main modules, each responsible for one aspect of conceptual design. The first module is used to identify the primary and secondary objectives determine product requirements. Primary objectives re requirements that the product must accomplish in order to be considered a success; secondary objectives are things that are desirable but are not necessary the product. These objectives are typically identified hrough brainstorming and stakeholders for the problem.

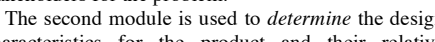
product and their relative design. The determination of the desig characteristics is once again carried out throug brainstorming sessions between design team member as well as meetings with ohter stakeholders and with elative importance of the design charattistics is determined using a pairwise comparison chart. In many design problems, the list of characteristics can be quite large. Using a pairwise comparison chart not only helps to make this task more manageable, but also encoulages discassion anongst tean menbers. rankings. The functional requirements are derived from the primary and secondary objectives and are

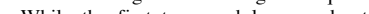

etter understanding of the design problem, the third module lets designers explore With the information gathered in the Identify an Determine modules, the design team now has a better understanding of what the boundaries of the trad space are. The Explore module lets the team use that in one of two ways: using trade space analysis techniques, or using a technique called ideation. The trade space analysis portion of the explore module is quite straightforward. The design team firs generates different options for each of the functiona requirements or subsystems in the final design. Thes diagram as shown in Figure 5. The design team the tries different combinations of options until they generate 5 to 10 plausible concepts that will go on to the final module for more detailed analysis.

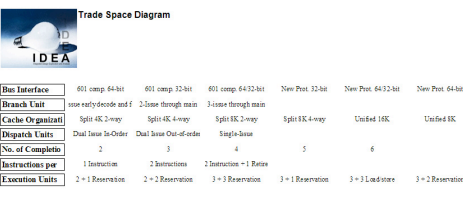

\section{Figure 5: IDEA Trade Space diagram}

The ideation option is a different way of explorin the trade space. In this case, each member of the characteristic. It is then the job of each team membe to generate concepts which optimize for their assigne characteristic at the expense of all others. The tean members then combine the various concepts together The final sodule of the IDEA procss is to andse

the resulting concepts in more detail to determin which is the most suitable for the design project an should move onto detailed design. This is done using a decision matrix as described in the previous section. A decision matrix is a perfect tool for this task since selecting amongst concepts is an example of decision matrix also allows for the participation of the entire design team when the various concepts are being rated. This allows multiple points of view to be represented in the final selection and improves the
odds that the best concept is identified. In addition, 
the decision matrix also utilizes the relative weights generated in the Determine module. If other determine those weight, hen their views will to expressed in the final concept selection.

\section{The IDEA Software Interface}

As defined in Section 3, the IDEA process brings ogether established methods to support conceptual designificant amount of "bookkeeping" This data recording and simple calculation can be easily and reliably computerised, which will increase the efficiency of the process because it frees the designers' time to focus on the real cognitive tasks. "iurthermore, computerisation of IDEA can provide an decision-making process

The IDEA software was created using an open source program called Compendium (hitp://www.compendiuminstitute.org) that The authors have extended Compendium by During the early stages of planning the software, the authors decided to use a graphical interface because of richly interconnected information of the kind one finds in engineering situation.

The main question was whether to use an existing graphics engine to power the interface, or whether to While a purpose built engine would have several advantages, the main disadvantage is that writing a graphics engine from scratch is very time consuming.
Thus, it was decided to find an existing engine that

would meet the needs of the interface.
After a search of several available options, the first is that Compendium is already graphically oriented, and the IDEA interface could be created with it without writing any code. Instead, Compendium uses a WYSIWYG interface to create databases and templates. This eased greatly the creation of the interface and reduced our development time. The an open source program, so it is both ubiquitis economical. Thus, using Compendium will help make IDEA available to a broad audience. Finally, Compendium is already being used in a wide variety of different settings. One of the most interesting uses has been at the Jet Propulsion Laboratory (JPL) in California where they use a custom interface built in
Compendium to operate the Mars Exploration Rovers currently on Mars [6]. That such a respected group

The software implements the structure IDEA as in . Special attention was given to make the our IDEA modules is assigned a separate region an colour on the home screen. Each of these region contains icons that represent the tasks that the desig cam must execute to complete that module. Doubleoutlines the tasks to be executed and provides acces task-specific tools. The home screen and sample lask windows are shown in Figures 6 and 7.

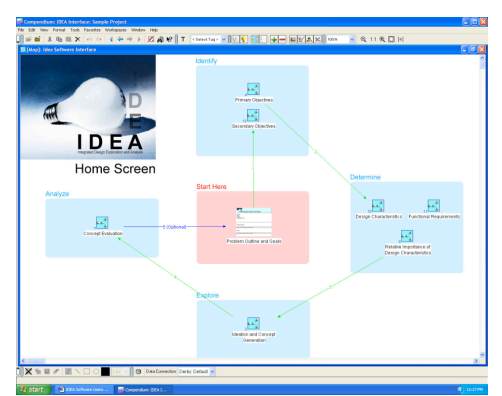

Figure 6: IDEA home screen

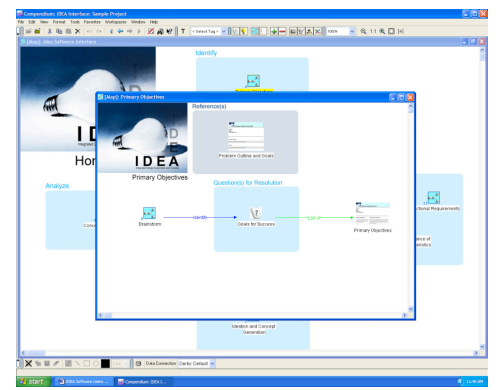

Figure 7: IDEA task window

In these figures, arrows connect the various icon and guide the user on what they should do next. In the
case of the task window, the arrows are labelled with suggested ways to complete specific tasks. For example, reading the thon and arrow text in Figure

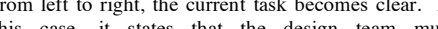
"Brainstorm to identify goals for success and a graphical structure is repeated in all of the tas windows to provide immediate guidance and familiar pattern to the user-

The brainstoming and primary objectives lists are each represented by their own icons, which can be
selected by a double-click. In the case of the brainstorming icon, a blank template is opened for the design team to use during a brainstorming session; double-clicking on the primary objectives icon opens template to record primary objectives. While the tea is still required to manually record the actual results of

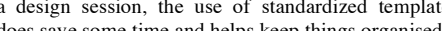
Two additional items of note in the IDEA interface are the Concept Evaluation Workbook and the Trade Space Analysis Tool. Recall that the overriding go of the IDEA software is to save time and increas efficiency. While the pairwise comparison and trade pace analysis techniques are very usefu, they are alo

In team must set up a matrix that crison chart, the large, and then calculate the weights of the desig characteristics based on the contents of that matrix. Both of these tasks can be quite difficult and prone error. The Concept Evaluation Workbook is used to chart, and as the design team conducts the pairwise comparisons, the workbook automatically calculates the relative importance of the design characteristics and displays them in real time both numerically an graphically. This not only saves time, but also improves accuracy since dhe con Fursterme the wo

book uses the information (t) generate an only has to fill in the appropriate ratings for each of the concepts and the workbook automatically calculates the aggregate scores, while simultaneously generating a graphical representation of the strength The Trade Space Analysis Too

The the generation of these diagrams can become quite tedious. Designers simply enter the various option available for hic funcional requironents and ope tool then automatically generates a trade space diagram. software to achieve its primary goal of allowing

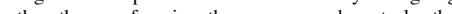
commonly associated with it.

\section{Case Studies}

In order to validate the effectiveness of the IDEA process three case studies were conducted. The three case struss we a bid ange of topics to not also to demonstrate its versatility in a wide range of different applications.

The first case study was based on work done during Masur's undergraduate thesis. It dealt with the desig of a sample-processing unit (SPU) for the Mars Science Laboratory mission. The purpose of the SPU Martion surface and process them down into a fine powder suitable for use in NASA-supplied scientific instruments. This problem was selected because it is good mechanical engineering problem, and becaus both authors are familiar with the original work While the act of performing the SPU design is of som from the results of the design.

The conceptual design in the original study was not done using any kind of standardized method. Because of this, only three different concepts were generated. pis podul sofware. The IDEA trade space analysi an initial screening step this number was reduced six, which is still double the number of concepts in the original study. The sheer increase in the $\mathrm{n}$ concepts generated demonstrates that the use of the DEA process has resulted in a much more thoroug explosed of the design space, which in turn has While a genterumber of cong the bese "concept. the analysis conducted with the decision matrix still resulted in the selection of the same concept as in the original study. Since the original study wa considered quite innovative and received high marks, this result seems to validate the effectiveness of IDEA. called th Spluncen Expors that is ued by schools to illustrate philosophies of law. It is a fictional problem set in the state of Newgarth in the year 4300 (7). In it, four men are trapped in a cave a forced to kill and consume one of their numbers to survive until rescue. They are subsequently rescued, and when they have sufficiently recovered, they are charged with mirder and cerchtally found guilty and 
it is the job of the reader to act as the head of Newgarth's lega he verdict.

The case is structured in such a way that there is no solving techne it is merely an exercise in problem second case study because it would provide a good contrast to the engineering problem of the first case study while also versatility of

One of the things that immediately became apparent was that not all of the tools and modules were applicable to the case. A problem such as this is more a subjective moral and ethical dilemma than an deemed improper to use tools such as trade space analysis and pairwise comparison to decide the the of the case. Most of the work was done in to gain a better understanding of the problem and to more clearly outline all of the facts.

While at first glance there may seem to be relatively few stakeholders in the outcome of the case, the analysis conducted using IDEA revealed that there are far more people with a vested interest in the outcome people have a drastic effect on the final verdict and to ignore them would lead to incomplete information and a less than optimal solution. Using the IDEA process it was determined that the men should be found guilty to uphold the laws of the state, but that their sentence should be commuted to account for the fact that they This is one of the options presented in the origit source, which once again confirms that the IDEA generate meaningful results. This, coupled with the fact that the use of IDEA leads to a better exploration of the possible options,
demonstrates that the process can be of use in demonstrates tha die process can problems other than just engineering.

dealt with the design of the for a number of reasons. Firstly, while it is engineering problem, it is in a different discipline than the first case study. This would once again demonstrate the versatility of the IDEA process in that it could be used in the many different engineering available on the design itself. There are several papers [8,9] available that describe the conceptual design of the actual designers in detail.

Much of the original work was done using a program called BRAT that was able to simulate the performance of the processor as it was being designed.
Many of the results of this were available which showed how the performance of the processor changed and the designers altered its characteristics. The use

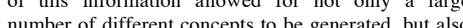
allowed each of these different concepts to be assigned rough performance values based on the analytic sults.

The application of the IDEA process allowed for the generation of eight different concepts for the fina design of the PowerPC 603. Each of these concept characteristics. Some sacrificed speed for lower power use and less heat generation, while othe concepts were the exact opposite and were designed for maximum performance at the expense of all othe characteristics. In the end, the authors identified concept that struck the best balance between all the design, the concept that was selected by the a was very similar to the actual one. This result yet gain proved that the IDEA process was generating meaningful results, while the large number of enerated concepts one again demonstrated tha forcing designers to analyze all aspects of the problen

\section{Conclusions}

It can be argued that conceptual design is the mos inportant phase of the engineering design process. rang process. The selection of an unsuitable concept ead to delays, budget overruns, and possibly projec cancellation. It is thus critical to conduct conceptu design in as thorough and logical a manner a possible.

Of great concern, however, is the fact that the most confusing because the early stages of design have very little hard information available and the vast array of different tools that designers have at the disposal can be very confusing. The purpose of the DEA process is to provide a standardized method for conceptual design that not only helps to remove cols re by press of the problem, but do sol of ensure a thorough exploration of the design space by forcing designers to look at many different aspects of design problem

The use of the software system enhances the usability and efficiency of the IDEA process since leverity all of its steng the studies demonstrate that these goals have been achieved. Not only has the use of IDEA lead to the case studies, but the use of the interface has a created permanent documentation which outlines not only what decisions were made, but why designes made them. The achievement of these goals has lead to an increase in the effectiveness of conceptual design which leads to the betterment of the engineering

\section{References}

[1] Wertz, James R. and Larson, Wiley J., Space Mission Analysis and Design $3^{\text {td }}$ Edition, Microcosm, El Segundo.

Using Customer Values", IEEF Transactions On Systems Man, and Cybernetics - Part C Applications and Reviews,

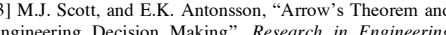
Design, 1999, pp. 218-228

[4] D.O. Anderson, Making Engineering Design Decisions, [5] SH. Zanakis, et al "Multipttibute De

Decision Making: Journal of Operational Research, Issue 107, 1998, pp. $507-$

6] Compendium Institute, "Compendium Community

html, (April 21 $1^{\mathrm{st}}, 2006$ )

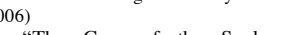
Explorers", Harvard Law Review, Vol. 62, 1949, pp.

[8] B. Burgess, et. al., "The PowerPC 603 Microprocessor", Vol. 37, No. 6, 1994, pp. 34-42

9] B. But Performance Analysis and Design Trade-Offs, Motorola and

\section{CKNOWLEDGEMENTS}

The authors graciously acknowledge the support of 